\title{
NO INFINITE DIMENSIONAL $P$ SPACE ADMITS A MARKUSCHEVICH BASIS
}

\author{
WILLIAM B. JOHNSON
}

Abstract. Theorem. Let $X$ be a Banach space. If $X$ is a Grothendieck space and $X$ admits a Markuschevich basis then $X$ is reflexive. This theorem is used to prove the conjecture of J. A. Dyer [1] stated in the title.

Recall that a Banach space $X$ is a Grothendieck space if every weak* convergent sequence in $X^{*}$ is weakly convergent. $X$ is a $P$ space if $X$ is complemented in every Banach space which contains it as a subspace. ${ }^{1}$ Since a complemented subspace of a Grothendieck space is a Grothendieck space and since every $P$ space can be embedded in the Grothendieck space $m(T)$ for a suitable set $T$, every $P$ space is a Grothendieck space. Infinite dimensional $P$ spaces are nonreflexive, so Dyer's conjecture is a consequence of our theorem.

Proof of the Theorem. Suppose that $\left\{x_{i}, f_{i}\right\}_{i \in I}$ is a Markuschevich basis for the Grothendieck space $X$; i.e., $\left\{x_{i}, f_{i}\right\}_{i \in I}$ is a biorthogonal collection in $\left(X, X^{*}\right)$ such that $\left\{x_{i}\right\}_{i \in I}$ is fundamental in $X$ and $\left\{f_{i}\right\}_{i \in I}$ is total over $X$. Let $Y$ be the norm closure in $X^{*}$ of the linear span of $\left\{f_{i}\right\}_{i \in I}$ and let $B$ be the closed unit ball of $Y$.

To show that $X$ is reflexive it is sufficient to show that $Y$ is reflexive. (Indeed, $Y$ is total over $X$ so that $Y$ is weak ${ }^{*}$ dense in $X^{*}$. If $B$ is weakly compact, ${ }^{2}$ then $B$ is weak* compact, so that it follows from the Krein-Smulian theorem that $Y$ is weak ${ }^{*}$ closed and hence $Y=X^{*}$.) By Eberlein's theorem, we need to show only that $B$ is weakly sequentially compact.

Let $\left\{y_{n}\right\}_{n=1}^{\infty}$ be a sequence in $B$. Since each $y_{n}$ is the norm limit of a sequence from the linear span of $\left\{f_{i}\right\}_{i \in I}$, it follows that for each $n$, the set $A_{n}=\left\{i \in I: y_{n}\left(x_{i}\right) \neq 0\right\}$ is countable and thus $\bigcup_{n=1}^{\infty} A_{n}$ is countable. A standard diagonalization argument shows that there is an increasing sequence $\{P(n)\}_{n=1}^{\infty}$ of positive integers such that $\lim _{n \rightarrow \infty} y_{P(n)}\left(x_{i}\right)$ exists for each $i \in I$. Since $\left\{y_{n}\right\}_{n=1}^{\infty}$ is equicontinuous

Presented to the Society, August 25, 1970 under the title Existence theorems for Markuschevich bases in Banach spaces; received by the editors February 24, 1970.

AMS 1969 subject classifications. Primary 4610.

Key words and phrases. Markuschevich basis, complete biorthogonal systems, $P$ spaces, injective Banach spaces, Grothendieck spaces.

${ }^{1}$ For the basic facts concerning $P$ spaces see [3]. The most interesting nonreflexive Grothendieck spaces are discussed in [2].

${ }^{2}$ Since the weak topology on $Y$ by $Y^{*}$ is the relativisation to $Y$ of the weak topology on $X^{*}$ by $X^{* *}$, there is no ambiguity in discussing the weak topology on $Y$. 
on $X$ and $\left\{x_{i}\right\}_{i \in I}$ is fundamental in $X, \lim _{n \rightarrow \infty} y_{P(n)}(x)$ exists for each $x \in X$. That is, $\left\{y_{P(n)}\right\}_{n=1}^{\infty}$ is weak* convergent to, say, $y$ in $X^{*}$. Since $X$ is a Grothendieck space, $\left\{y_{P(n)}\right\}_{n=1}^{\infty}$ is weakly convergent to $y$. Finally, $y$ is in $Y$ (and hence in $B$ ) because the weak and norm closures in $X^{*}$ of the linear span of $\left\{f_{i}\right\}_{i \in I}$ are the same. Thus $B$ is weakly sequentially compact and the proof is complete.

\section{REFERENCES}

1. J. A. Dyer, Generalized bases in P-spaces (preprint).

2. A. Grothendieck, Sur les applications linéaires faiblement compactes d'espaces $d u$ type $C(K)$, Canad. J. Math. 5 (1953), 129-173. MR 15, 438.

3. J. Lindenstrauss, Extension of compact operators, Mem. Amer. Math. Soc. No. 48 (1964). MR 31 \#3828.

University of Houston, Houston, Texas 77004 